\title{
THE ASYMMETRIC DRIFT OF THE THICK DISK POPULATION
}

DEVENDRA OJHA, ANNIE C. ROBIN AND OLIVIER BIENAYMÉ Observatoire de Besançon, BP 1615, F-25010 Besançon, France

We investigate the kinematics of the thick disk population using photometric and astrometric sample surveys towards two opposite directions at intermediate latitude. A multivariate discriminant analysis (MDA) is used to distinguish the 'Thick Disk' from other populations with the help of the Besançon model of population synthesis. The data constrain the asymmetric drift of the thick disk population, which is found to be $40 \pm 10 \mathrm{~km} / \mathrm{s}$ and does not depend on the galactic radius.

The two data sets with 5-parameters (V, B-V, U-B, $\mu_{l}$ and $\left.\mu_{b}\right)$ towards the galactic centre $\left(l=3^{\circ}, b=47^{\circ}\right.$; Ojha et al. 1994b) and anticentre $(l=$ $167^{\circ}, b=47^{\circ}$; Ojha et al. 1994a) were used to derive the asymmetric drift of the thick disk population. Only stars with $14<\mathrm{V}<15.5$ and $\mathrm{B}-\mathrm{V}<0.8$ were selected for this purpose. We apply a multivariate discriminant analysis method to determine the best axis to discriminate the thick disk population from the thin disk and the halo. The resulting discriminant axis is a linear combination of the five parameters $\left(\mathrm{V}, \mathrm{B}-\mathrm{V}, \mathrm{U}-\mathrm{B}, \mu_{l}, \mu_{b}\right)$ and is dominated by the proper motion along the rotation $\left(\mu_{l}\right)$ and by the U-B color index due to metallicity differences between the thin disk, thick disk and halo.

To quantitatively estimate the adequacy of the models with various circular velocities, we applied Kolmogorov-Smirnov and $\chi^{2}$-tests to the distributions of model predicted stars and the observed one on the first discriminant axis. The analysis estimates the circular velocity of the thick disk to be $180 \pm 10 \mathrm{~km} / \mathrm{s}$. Assuming the Local Standard of Rest $\mathrm{V}_{L S R}=220 \mathrm{~km} / \mathrm{s}$, this gives an asymmetric drift of thick disk population of $40 \pm 10 \mathrm{~km} / \mathrm{s}$ with respect to the LSR. We obtained a unique value in both directions, showing that no radial gradient seems to occur on a base of $3 \mathrm{kpc}$ around the Sun.

\section{References}

Ojha D.K., Bienaymé O., Robin A.C., Mohan V., 1994a, A\&A 284, 810

Ojha D.K., Bienaymé O., Robin A.C., Mohan V., 1994b, A\&A 290, 771 